\title{
Physical Activity and Serum Cytokines Levels in Depressed In- dividuals - Gender Differences
}

\section{Carolina David Wiener, Fernanda Fedrotti, Jean Pierre Oses, Karen Jansen, Diogo Rizzato Lara, Ricardo Azevedo da Silva and Jeronimo Costa Branco*}

Department of Health and Life Sciences, Centro Franciscan University - UNIFRA, Brazil

*Corresponding author: Jeronimo Costa Branco, Doctor of Health and Behavior, Lecturer, Franciscan University Center, Physiotherapy Course; Master in Health and Life Sciences, Centro Franciscan University - UNIFRA, Brazil, Tel: 5553981090937, E-mail: jeronimobranco@hotmail.com

\begin{abstract}
Background: The efficacy of physical activity on the reduction of depressive symptoms may be attributed to its impact over neuroinflammatory mechanisms. The aim of this study is to investigate the relationship between habitual practice of physical activities and serum cytokines levels in individuals diagnosed with depression.

Methods: Cross-sectional study nested within a population-based community study from which depressed adults aged 18 to 35 years were selected. The presence of depression was verified through the Mini International Neuropsychiatric Interview 5.0 (M.I.N.I.). The practice of leisure-time physical activity was evaluated using the International Physical Activity Questionnaire 8.0 (IPAQ). Serum levels of the cytokines IL-6, IL-10, and TNF- $\alpha$ were analyzed through the ELISA test.

Results: Sample was composed of 142 depressed individuals. Cytokines were not associated with any independent variables stratified by gender. The group of active men presented increased IL-10 serum levels $(p=0.032)$, decreased TNF- $\alpha$ levels $(p=0.014)$, and decreased fraction of TNF- $\alpha$ / IL-10 ratio ( $p=0.001)$, when compared to inactive men. There was no difference in serum cytokines levels and leisure-time physical activity in women.

Conclusions: These findings may validate the importance of habitual leisure-time physical activity amongst depressed individuals and indicate that physical activity may modulate inflammatory paths involved in the physiopathology of depression.
\end{abstract}

\section{Keywords}

Physical Activity, Depression, Cytokines, Inflammation

\section{Background}

Major Depression Disorder (MDD) is characterized by persistent low mood and diminished ability of feeling satisfaction and pleasure; it significantly reduces quality of life and leads to economic and social impairments. The prevalence of MDD in general population is $17 \%$ [1]. Over the last few years, important developments regarding the treatment of depression have been made. However, a portion of depressed patients is refractory to traditional treatments, which may lead to the chronicity of the disorder and lead to suicide in severe cases [2].

The mechanisms involved in this disorder are not yet completely recognized. There are psychological, genetic and environmental factors that are known to be involved in the vulnerability for depression. Studies show that depression produces an increase in proinflammatory cytokines levels [3,4]. These alterations will have consequences regarding the suppression of neurotrophic support that is related to atrophy, degeneration, and loss of excitatory neurotransmitters liberation [5]. The reduction of neurotrophic support in some brain areas during depression has also been associated with the dopamine, noradrenaline and serotonin systems, which are intimately related to the onset and maintenance of depressive episodes [6]. Elevated levels of pro-inflammatory cytokines and positive proteins activated by the immunological system have been associated with depression $[7,8]$. 
In addition, studies report the existence of a common path between physical activity and inflammatory cytokines. This similarity of mechanisms allows speculation that physical activity may modulate the inflammatory paths that may be involved in the physiopathology of depression [9].

The efficacy of physical activity on the reduction of depressive symptoms may be attributed to its impact over neurobiological and neuroinflammatory mechanisms [9]. Physical exercise and the practice of non-structured physical activities $[10,11]$ have been hypothesized as neuromodulators of anti- and pro-inflammatory cytokines, reducing IL- $6[10,11]$ and TNF- $\alpha[11]$ levels and increasing the concentration of IL-10 $[12,13]$. Thus, the aim of this study is to investigate the relationship between the habitual practice of leisure-time activities and the serum levels of IL-6, IL-10, and TNF- $\alpha$ in individuals diagnosed with depression, considering the difference between genders.

\section{Method}

This is a cross-sectional study nested within population-based community sample, from which 1953 young adults aged 18 to 35 -years-old from a southern city in Brazil were evaluated. Individuals were considered eligible for the study if they had a diagnosis of depression and did not use psychoactive substances. This study was approved by the Catholic University of Pelotas Ethics Committee (2010/15).

The participants received information about the study and had to sign informed consent. Afterwards, the participants answered to a questionnaire with questions concerning socio-demographic information (gender, age, schooling and ethnicity), use of psychoactive substances (medication, tobacco, alcohol, marijuana and cocaine). Socioeconomic evaluation was carried out using the IEN criteria (National Economic Index, Brazil - Índice Econômico Nacional, Brasil), which is based on the accumulation of material assets and on the schooling of the head of the household. These criteria generate a continuous variable which was presented in textiles [14]. For the propose of this present study, we selected men and women that been diagnosed with depression and did not use psychoactive substances.

The diagnosis of depression was verified through the standardized clinical interview Mini International Neuropsychiatric Interview 5.0 (M.I.N.I.) [15]. This instrument is widely used in clinical and research practice to classify the interviewees accordingly to the DSM-IV and ICD-10 diagnostic criteria.

The leisure-time physical activity was evaluated using the International Physical Activity Questionnaire 8.0 (IPAQ). The total score of physical activity was measured as suggested by Hallal, et al. [16] by adding the number of minutes of moderate activity plus the minutes of vigorous activity, and finally, plus the minutes of walk- ing activity - all for at least 10 uninterrupted minutes. For the purposes of this study, the participants who reported over 150 minutes of weekly physical activity were considered as active individuals. This criterion is in accordance with the current recommendations for the practice of physical activity [16].

For the cytokines levels measurements, 10 milliliters of blood were withdrawn from each subject by venipuncture into an anticoagulant-free vacuum tube after the interview. The blood was immediately centrifuged at $3,500 \mathrm{rpm}$ for $15 \mathrm{~min}$, and serum was kept frozen at $-80{ }^{\circ} \mathrm{C}$ until analysis. Serum IL-6, IL-10, TNF- $\alpha$ levels were measured using a commercial immunoassay kit (DuoSet ELISA Development, R\&D Systems, Inc., USA). Levels were expressed in $\mathrm{pg} / \mathrm{mL}$. The ratio of pro and anti-inflammatory cytokines (IL-6/IL-10 and TNF- $\alpha /$ IL-10 ratio) could provide an index for the balance between pro- and anti-inflammatory status, and these ratios are considered an index of net pro-inflammatory activity.

Statistical analyzes were performed in the software SPSS 21 and GraphPad Prism 6.0. The variables age, years of study, and Body Mass Index (BMI) were presented using mean and standard-deviation values, whereas color of the skin, economic indicator and gender were presented using relative and absolute frequencies. Serum levels of inflammatory cytokines did not present Gaussian distribution and were presented using median and interquartile range.

The Student $t$ test was used to verify mean differences among the variables age, years of study, and BMI between men and women. The proportion differences between color of the skin and IEN were tested in relation to gender using the chi-square test. The non-parametric tests Mann-Whitney and Kruskal-Wallis were used to verify the association between independent variables and practice of physical activity and cytokine levels. The results with $p$-values of $\leq 0.05$ were considered statistically significant.

\section{Results}

The population-based sample was composed of 1953 individuals. Of these, 880 participants were male and 1073 were female. Men and Women were eligible for the present study if they had been diagnosed with depression and did not use psychoactive substances. Thus, the present sample consisted of 142 individuals. Of these, 57 participants were male and 85 were female.

Concerning sample characteristics, men and women differed regarding age $(p=0.047)$, economic indicator $(p=0.009)$, and IPAQ score $(p=0.044)$ (Table 1). Consequently, the hypothesis tests were stratified by gender.

None of the independent variables (age, years of study, color of the skin, economic indicator and BMI) was associated with serum cytokine levels (IL-6 and IL10 and TNF- $\alpha$ ), after stratification by gender. [Data not presented]. 
Table 1: Sample characteristics in men and women with depression.

\begin{tabular}{|c|c|c|c|}
\hline Variables & Depressed men & Depressed women & P-value \\
\hline $\mathrm{Age}^{\mathrm{a}}$ & $25.61 \pm 5.36$ & $27.41 \pm 5.15$ & 0.047 \\
\hline Years of study ${ }^{a}$ & $10.60 \pm 3.64$ & $9.60 \pm 3.70$ & 0.116 \\
\hline Color of the skin ${ }^{b}$ & & & 0.554 \\
\hline Caucasian & $43(42.2 \%)$ & $59(57.8 \%)$ & \\
\hline Non-Caucasian & $14(35.0 \%)$ & $26(65.0 \%)$ & \\
\hline NEI ${ }^{*} \mathbf{b}$ & & & 0.009 \\
\hline Low & $12(25.5 \%)$ & $35(74.5 \%)$ & \\
\hline Medium & $18(38.3 \%)$ & $29(61.7 \%)$ & \\
\hline High & $26(56.5 \%)$ & $20(43.5 \%)$ & \\
\hline $\mathrm{BMI}^{\mathrm{a}}$ & $25.92 \pm 4.49$ & $26.98 \pm 5.65$ & 0.242 \\
\hline Practice of physical activity ${ }^{b}$ & & & 0.044 \\
\hline Yes & $13(61.9 \%)$ & $8(38.1 \%)$ & \\
\hline No & $44(36.4 \%)$ & $77(63.6 \%)$ & \\
\hline Interleukin $6^{c}$ & $17.57(12.54-28.59)$ & $17.53(11.93-26.35)$ & 0.726 \\
\hline Interleukin $10^{c}$ & $55.74(37.32-86.00)$ & $57.37(45.91-74.78)$ & 0.170 \\
\hline Tumor Necrosis Fator $-\alpha^{c}$ & $19.21(11.89-36.10)$ & $15.52(11.38-23.67)$ & 0.148 \\
\hline Total & 57 & 85 & \\
\hline
\end{tabular}

aMean and standard-deviation; ${ }^{b} \mathrm{Absolute}$ and relative frequencies; 'Median and interquartile intervals.

*Indicador Econômico Nacional - Brasil (National Economic Indicator - Brazil).
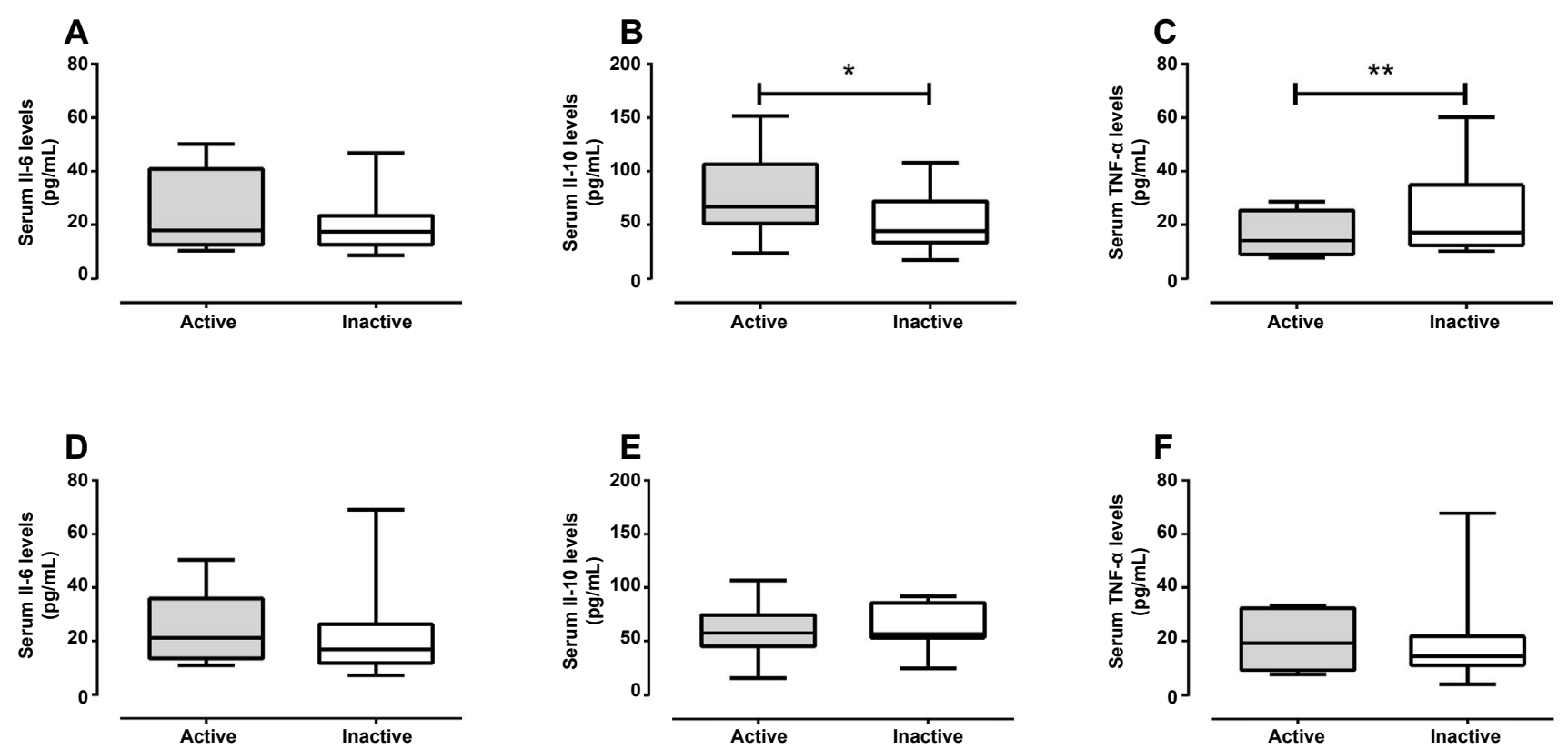

Figure 1: Serum cytokine levels in active and inactive men (Figure A, B, and C) and women (Figure D, E, and F).

Serum IL-6 levels did not significantly differ regarding habitual practice of leisure-time physical activity in men $(p=0.546$, Figure $1 A)$. Active men presented increased serum IL-10 levels ( $p=0.032$; Figure $1 B$ ) and decreased serum TNF- $\alpha$ levels ( $p=0.014$; Figure $1 C$ ), when compared to inactive men. Active women did not differ regarding serum IL-6 $(p=0.270$, Figure $1 D)$, IL-10 $(p=$ 0.449 , Figure $1 E)$, and TNF- $\alpha(p=0.417$, Figure $1 F)$ levels, when compared to the group of women that did not perform habitual leisure-time physical activity.

For the purpose of verifying the relationship between the pro-inflammatory cytokine (IL- 6 and TNF- $\alpha$ ) and the anti-inflammatory cytokine (IL-10), a ratio of these cytokine interleukins levels was performed for each individual. For men regarding inflammatory status indicated by the pro- and anti-inflammatory cytokine ratio, no difference was observed in the IL-6/IL-10 ratio $(p=0.607$, Figure $2 A$ ) whereas, there was a significant decrease in the TNF- $\alpha /$ IL-10 ratio ( $p=0.001$, Figure $2 B)$. The ratio of pro- and anti-inflammatory cytokine did not differ between active and inactive women (IL-6/IL-10: $p=0.542$, Figure 2C e TNF- $\alpha$ /IL-10: $p=0.761$, Figure 2D).

\section{Discussion}

In this study, depressed men who regularly practice physical activities presented increased serum levels of the anti-inflammatory cytokine IL-10, and significantly decreased serum levels of the pro-inflammatory cytokine TNF- $\alpha$. Moreover, men presented increased inflammatory state, specifically regarding TNF- $\alpha /$ IL-10. 

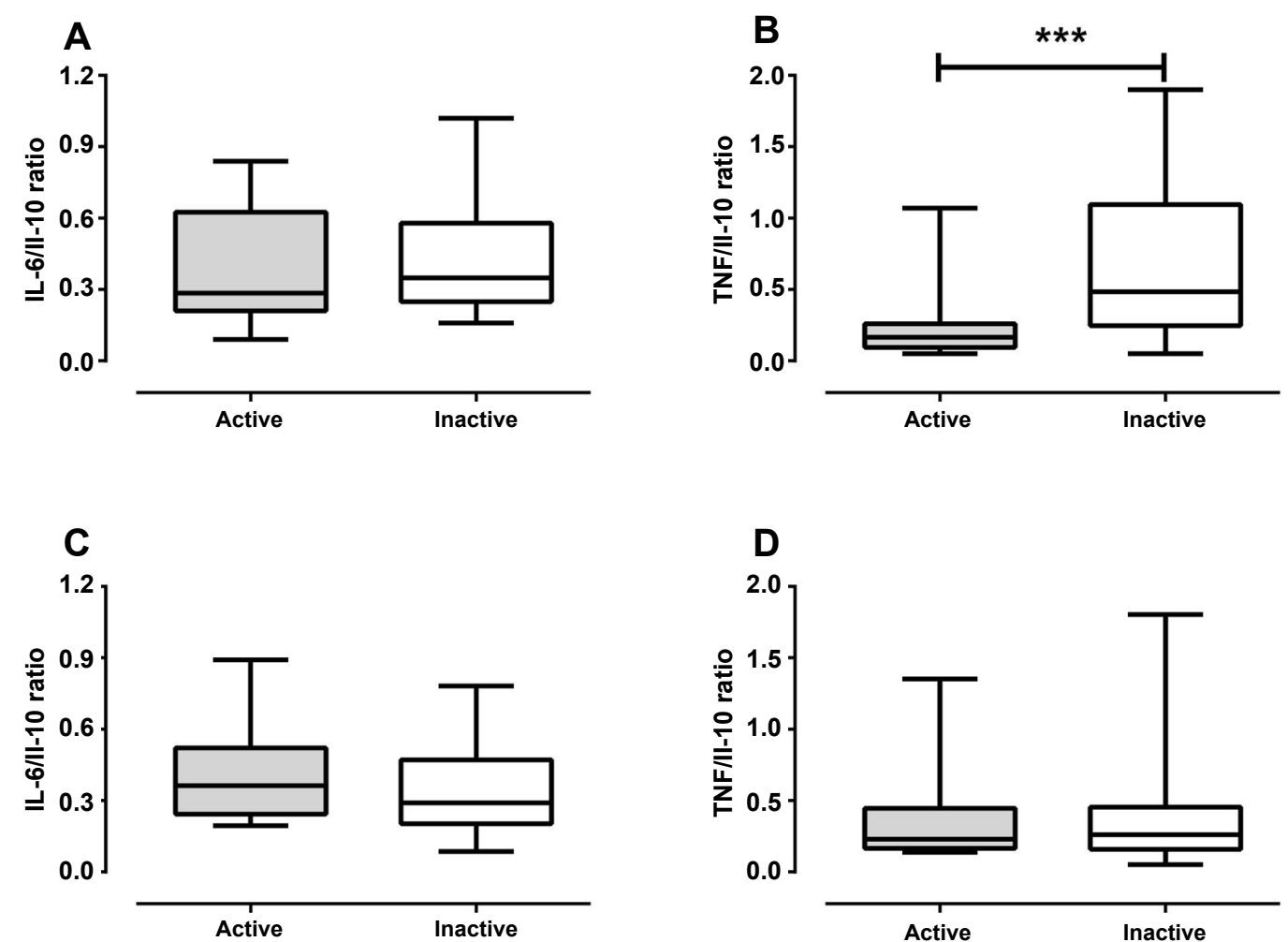

Figure 2: Ratio of pro- and anti-inflammatory cytokine in active and inactive men (Figure A and B) and women (Figure C and D).

Concerning women, the practice of physical activities did not interfere with the serum levels of the evaluated cytokine.

There is a bidirectional connection between the Central Nervous System (CNS) and the immunological system [17]. The CNS regulates the immunological system through neuronal and neuroendocrinological paths. In turn, the immunological system signals the brain through neuronal and humoral paths, and through the action of cytokines [18]. The understanding of the interaction between the immunological and neuroendocrinological systems demonstrated that it performs an important role in many disorders, including neurological and psychiatric disorders [19]. Cytokines and other immunological factors perform a key role in brain modulation; however, chronic exposure to pro-inflammatory cytokines may damage neuronal plasticity and contribute to cognitive disorders and mood alterations $[7,20]$. Thus, biomarkers of inflammatory activity, such as proand anti-inflammatory cytokines, have been correlated with depression $[8,20,21]$ being found an increase in pro-inflammatory cytokines and the decrease in the anti-inflammatory in depressives [21,22].

On the scope of mental health, a great group of individuals with mental disorders, including depression, may benefit from the practice of physical activity $[23,24]$. Physical exercise may modulate inflammatory paths involved in the physiopathology of depression and it is a modality of treatment that avoids possible adverse effects resulting from pharmacological inter- ventions [24]. Meta-analysis studies reveal an inverse relationship between physical exercise and depression $[24,25]$. In this context, the practice of physical activity has shown to benefit the treatment of psychiatric disorders with aims of reducing depressive symptoms. It may also have neuropsychological and neurobiological implications, including the inflammatory system $[26,27]$. Our study revealed an increase in the anti-inflammatory cytokines levels and a decrease in the pro-inflammatory cytokines levels in depressed men who practice physical activities, which indicates that this practice modulates inflammatory paths and may help in the treatment of depression.

The musculoskeletal work performed during the exercise is believed to act like an endocrinological organ, producing, releasing and counterbalancing cytokines [28-32]. IL-6 has a fundamental role in the anti-inflammatory process resulting from exercise, and it presents both pro and anti-inflammatory characteristics [33]. One review study from Benatti and Pedersen, 2014, reported that muscle contraction produces myokines that, alongside other peptides, induce an anti-inflammatory response. Thus, there is an exacerbated IL- 6 secretion in the beginning of the exercise which consequently increases the liberation of IL-1 and IL-10 and inhibits the TNF- $\alpha$ production during the exercise [34]. Studies have found that individuals who perform physical exercises present improved concentrations of inflammatory markers, resulting in decreased expression of TNF- $\alpha$ $[12,35]$ as well as an increase in the IL-10 at the hippocampus [12]. These evidences may justify our results 
of decreased serum TNF- $\alpha$ levels concentration and increased IL-10 levels in depressed men who practiced leisure-time physical activities.

We did not find significant differences regarding the serum IL-6 levels in both active men and women. II-6 is the first cytokine with pro-inflammatory characteristics to be released in the beginning of the exercise. On the other hand, there is a constant decrease in this cytokine concentration during the period of recovery from the exercise. These alterations provoked by physical exercise may justify our results $[34,36,37]$. Similar results have been found by other authors [11,38], who did not find a significant association between serum IL-6 levels and the practice of physical activity.

In addition, the practice of physical activity indicates a positive effect on inflammatory balance [39]. Significant alterations on pro- and anti-inflammatory balance (TNF- $\alpha /$ IL-10) were found amongst depressed men in this sample. This finding is in agreement with literature including studies that evaluated the effect of the practice of physical activity and inflammatory balance (pro and anti-inflammatory) on serum concentrations [39] and on animal hippocampus [12].

In our study, the analyzed cytokines levels (IL-6, IL10 and TNF- $\alpha$ ) did not present significant differences regarding the practice of leisure-time physical activities in women. In contrast to our results, Pereira [13] found that women in better physical conditions presented lower IL- 6 and TNF- $\alpha$ concentration and increased IL-10 concentration [13]. Conversely, other studies, including our own, did not find a significant association between physical activity and cytokine in women $[11,40]$. One possible explanation for the different results regarding gender may be related to hormonal differences that interfere with the inflammatory profile [41] and to the fact that women present physiologically increased fat concentration, which releases a high concentration of pro-inflammatory cytokines [42]. These may be the reasons why physical activity did not alter cytokine levels in the feminine gender in the present study.

The limitation of this work is the small sample size due to eligibility criteria [individuals in current unipolar depressive episode who did not use current psychotropic medication]. Nonetheless, sample selection originated from a population-based study, which guarantees representativeness of the sample. Moreover, literature lacks studies investigation the relationship between physical activity and serum cytokines levels in depressed patients. Results suggest physical activity may be acting as a modulator of the inflammatory paths involved in the physiopathology of depression.

\section{Conclusion}

The findings of this study may legitimate the importance of habitual leisure-time physical activities amongst depressed men, since active men presented a higher con- centration of anti-inflammatory cytokine (IL-10) and a lower concentration of pro-inflammatory cytokine (TNF- $\alpha$ ). Nonetheless, we did not observe significant differences regarding cytokine serum levels in women. More studies are needed to clarify the effect of physical activity on the physiopathology of depression and help its treatment.

\section{References}

1. Schimidt HD, Shelton RC, Duman RS (2011) Functional biomarkers of depression diagnosis, treatment, and pathophysiology. Neuropsychopharmacology 36: 2375-2394.

2. Tadic A, Wagner S, Gorbulev S, Dahmen N, Hiemke C, et al. (2011) Peripheral blood and neuropsychological markers for the onset of action of antidepressant drugs in patients with major depressive disorder. BMC Psychiatry 11: 16.

3. Eyre H, Baune BT (2012) Neuroplastic changes in depression: a role for the immune system. Psychoneuroendocrinology 37: 1397-1416.

4. Post RM, Altshuler LL, Frye MA, Suppes T, McElroy S, et al. (2006) New findings from the Bipolar Collaborative Network: clinical implications for therapeutics. Curr Psychiatry Rep 8: 489-497.

5. Jovanovic JN, Czernik AJ, Fienberg AA, Greengard P, Sihra TS (2000) Synapsins as mediators of BDNF-enhanced neurotransmitter release. Nat Neurosci 3: 323-329.

6. Garcia C, Chen MJ, Garza AA, Cotman CW, Russo-Neustadt A (2003) The influence of specific noradrenergic and serotonergic lesions on the expression of hippocampal brain-derived neurotrophic factor transcripts following voluntary physical activity. Neuroscience 119: 721-732.

7. Loftis JM, Huckans M, Morasco BJ (2010) Neuroimmune mechanisms of cytokine-induced depression: current theories and novel treatment strategies. Neurobiol Dis 37: 519-533.

8. Voorhees JL, Tarr AJ, Wohleb ES, Godbout JP, Mo X, et al. (2013) Prolonged restraint stress increases IL-6, reduces IL-10, and causes persistent depressive-like behavior that is reversed by recombinant IL-10. PLoS One 8: e58488.

9. Eyre HA, Baune BT (2014) Assessing for unique immunomodulatory and neuroplastic profiles of physical activity subtypes: A focus on psychiatric disorders. Brain Behav Immun 39: 42-55.

10. Craft LL, Guralnik JM, Ferrucci L, Liu K, Tian L, et al. (2008) Physical activity during daily life and circulating biomarker levels in patients with peripheral arterial disease. Am J Cardiol 102: 1263-1268.

11. Hamer M, Steptoe A (2008) Walking, vigorous physical activity, and markers of hemostasis and inflammation in healthy men and women. Scand J Med Sci Sports 18: 736-741.

12. Gomes da Silva S, Simões PS, Mortara RA, Scorza FA, Cavalheiro EA, et al. (2013) Exercise-induced hippocampal anti-inflammatory response in aged rats. J Neuroinflammation 10: 61.

13. Pereira DS, Mateo EC, de Queiroz BZ, Assumpção AM, Miranda AS, et al. (2013) TNF- $\alpha$, IL6, and IL10 polymorphisms and the effect of physical exercise on inflammatory parameters and physical performance in elderly women. Age (Dordr) 35: 2455-2463.

14. Barros AJD, Victora C (2005) A nationwide wealth score based on the 2000 Brazilian demographic census. Rev Saúde Pública 39: 523-529.

15. Amorim P (2000) Mini International Neuropsychiatric Inter- 
view (MINI): Validação de entrevista breve para diagnóstico de transtornos mentais. Rev Bras Psiquiatr 22: 106-115.

16. Hallal PC, Gomez LF, Parra DC, Lobelo F, Mosquera J, et al. (2010) Lessons learned after 10 years of IPAQ use in Brazil and Colombia. J Phys Act Health 7: S259-S264.

17. Sternberg EM (2000) Interactions between the immune and neuroendocrine systems. Prog Brain Res 122: 35-42.

18. Webster JI, Tonelli L, Sternberg EM (2002) Neuroendocrine regulation of immunity. Annu Rev Immunol 20: 125-163.

19. Carney RM, Freedland KE (2003) Depression, mortality, and medical morbidity in patients with coronary heart disease. Biol Psychiatry 54: 241-247.

20. Blume J, Douglas SD, Evans DL (2011) Immune suppression and immune activation in depression. Brain Behav Immun 25: 221-229.

21. Dhabhar FS, Burke HM, Epel ES, Mellon SH, Rosser R, et al. (2009) Low serum IL-10 concentrations and loss of regulatory association between IL- 6 and IL-10 in adults with major depression. J Psychiatr Res 43: 962-969.

22. Dantzer R, O'Connor JC, Freund GG, Johnson RW, Kelley $\mathrm{KW}$ (2008) From inflammation to sickness and depression: when the immune system subjugates the brain. Nat Rev Neurosci 9: 46-56.

23. Ho CW, Chan SC, Wong JS, Cheung WT, Chung DW, et al. (2014) Effect of aerobic exercise training on chinese population with mild to moderate depression in Hong Kong. Rehabil Res Pract 2014: 627376.

24. Cooney GM, Dwan K, Greig CA, Lawlor DA, Rimer J, et al. (2013) Exercise for depression. Cochrane Database of Syst Rev.

25. Callaghan $P$ (2004) Exercise: a neglected intervention in mental health care? J Psychiatr Ment Health Nurs 11: 476-483.

26. Dunn AL, Trivedi MH, Kampert JB, Clark CG, Chambliss $\mathrm{HO}$ (2005) Exercise treatment for depression: efficacy and dose response. Am J Prev Med 28: 1-8.

27. Ströhle A (2009) Physical activity, exercise, depression and anxiety disorders. J Neural Transm 116: 777-784.

28. Pedersen BK, Febbraio MA (2008) Muscle as an endocrine organ: focus on muscle-derived interleukin-6. Physiol Rev 88: $1379-1406$.

29. Ostrowski K, Schjerling P, Pedersen BK (2000) Physical activity and plasma interleukin- 6 in humans--effect of intensity of exercise. Eur J Appl Physiol 83: 512-515.
30. Petersen AM, Pedersen BK (2005) The anti-inflammatory effect of exercise. J Appl Physiol (1985) 98: 1154-1162.

31. Mathur N, Pedersen BK (2008) Exercise as a mean to control low-grade systemic inflammation. Mediators Inflamm 2008: 109502.

32. Nielsen AR, Pedersen BK (2007) The biological roles of exercise-induced cytokines: IL-6, IL-8, and IL-15. Appl Physiol Nutr Metab 32: 833-839.

33. Pedersen BK, Steensberg A, Schjerling P (2001) Muscle-derived interleukin-6: possible biological effects. J Physiol 536: 329-337.

34. Muñoz-Cánoves $P$, Scheele $C$, Pedersen BK, Serrano AL (2013) Interleukin-6 myokine signaling in skeletal muscle: a double-edged sword? FEBS J 280: 4131-4148.

35. Chennaoui M, Drogou C, Gomez-Merino D (2008) Effects of physical training on IL-1beta, IL-6 and IL-1ra concentrations in various brain areas of the rat. Eur Cytokine Netw 19: 8-14.

36. Smith JK, Dykes R, Douglas JE, Krishnaswamy G, Berk S (1999) Long-term exercise and atherogenic activity of blood mononuclear cells in persons at risk of developing ischemic heart disease. JAMA 281: 1722-1727.

37. Benatti FB, Pedersen BK (2015) Exercise as an anti-inflammatory therapy for rheumatic diseases-myokine regulation. Nat Rev Rheumatol 11: 86-97.

38. Ko IG, Choi PB (2013) Regular exercise modulates obesity factors and body composition in sturdy men. J Exerc Rehabil 9: 256-262.

39. Santos RV, Viana VA, Boscolo RA, Marques VG, Santana MG, et al. (2012) Moderate exercise training modulates cytokine profile and sleep in elderly people. Cytokine 60: 731-735.

40. García JJ, Bote E, Hinchado MD, Ortega E (2011) A single session of intense exercise improves the inflammatory response in healthy sedentary women. J Physiol Biochem 67: 87-94.

41. You T, Berman DM, Ryan AS, Nicklas BJ (2004) Effects of hypocaloric diet and exercise training on inflammation and adipocyte lipolysis in obese postmenopausal women. J Clin Endocrinol Metab 89: 1739-1746.

42. Taghian F, Esteki Ghashghaei F, Badami R, Esteki Ghashghaei $S$ (2011) Comparison the effect of one session submaximal exercise on plasma levels of IL6 and TNF- $a$ in obese and non-obese women. ARYA Atheroscler 6: 153156. 